\title{
NAOTO KAIO
}

\section{SHUNJI OSAKI}

\section{Extended block replacement models}

Revue française d'automatique, d'informatique et de recherche opérationnelle. Recherche opérationnelle, tome 18, n ${ }^{\circ} 1$ (1984), p. $59-70$.

<http://www.numdam.org/item?id=RO_1984_18_1_59_0>

(C) AFCET, 1984, tous droits réservés.

L'accès aux archives de la revue « Revue française d'automatique, d'informatique et de recherche opérationnelle. Recherche opérationnelle » implique l'accord avec les conditions générales d'utilisation (http://www.numdam.org/ legal.php). Toute utilisation commerciale ou impression systématique est constitutive d'une infraction pénale. Toute copie ou impression de ce fichier doit contenir la présente mention de copyright.

\section{Numdam}

Article numérisé dans le cadre du programme

Numérisation de documents anciens mathématiques

http://www.numdam.org/ 


\title{
EXTENDED BLOCK REPLACEMENT MODELS ( ${ }^{*}$ )
}

\author{
by Naoto KaIo $\left({ }^{1}\right)$ and Shunji OsaKI $\left({ }^{2}\right)$
}

\begin{abstract}
In this paper we discuss an extended block replacement model in which after a preventive maintenance a system is not always as good as new. We apply, as a criterion of optimality, the expected total discounted cost for an infinite time span, and obtain the optimum interval between preventive maintenances minimizing that expected cost. We apply principle of optimality for analysis. We first discuss the model with one failure mode, and then the model with several failure modes as the extension of the first one.
\end{abstract}

Keywords: Block replacement, principle of optimality, minimal repair, optimization.

Résumé. - Nous discutons dans cet article une extension du modèle de remplacement par blocs dans laquelle le système, après une main tenance préventive, n'est pas toujours aussi bon que s'il était neuf. Le critère d'optimalité est l'espérance du cô̂t total actualisé pour un intervalle de temps infini. Nous obtenons l'intervalle de temps entre maintenances préventives qui minimise le critère précédent. Nous appliquons le principe d'optimalité pour l'analyse. Nous discutons d'abord le modèle à une défaillance, puis le modèle à plusieurs défaillances comme extension du premier.

\section{INTRODUCTION}

Block replacement models and their modifications have been discussed by many authors. In a block replacement model, a one-unit system is considered and the unit is replaced at failure and exchanged at a prespecified time, respectively (e. g., see Barlow and Hunter [1], Barlow and Proschan [2,3] and Berg and Epstein $[5,6])$. Since the age of the system does not need to be recorded in the block replacement model, the block replacement model is, in general, easier to apply to the practical situations than an age replacement model, in which a one-unit system is considered and the unit is replaced at failure or exchanged at a prespecified age, whichever occurs first (e. g., see Barlow and Proschan [2, 3] and Kaio and Osaki [7]). However, the block replacement model is more wasteful than the age one, since the unit may be exchanged soon after a

$\left(^{*}\right)$ Received May 1983.

(1) Department of Management Science, Hiroshima Shudo University, Hiroshima 731-31, Japan.

(2) Department of Industrial and Systems Engineering, Hiroshima University, HigashiHiroshima 724, Japan.

R.A.I.R.O. Recherche opérationnelle/Operations Research, 0399-0559/1984/59/\$ 5.00

(C) AFCET-Bordas-Dunod 
replacement in the block one. Modifications of the block replacement model have been proposed to overcome its fault.

Barlow and Hunter [1] consider the block replacement model, in which the system is maintened preventively and periodically and the failed system before a preventive maintenance undergoes a minimal repair, by which the system failure rate is undisturbed. After a preventive maintenance the system is assummed to become as good as new. However, in practice, that assumption is too strong and after a preventive maintenance the system is not always as good as new. That is, after a preventive maintenance the failure rate is smaller than, or equal to, or greater than one before a preventive maintenance. Thus, we extend the above model by Barlow and Hunter [1] to the block replacement model in which after a preventive maintenance the system is not always as good as new.

We apply, as a criterion of optimality, the expected total discounted cost for an infinite time span, especially with an exponential type discount rate, and obtain the optimum interval between preventive maintenances minimizing that expected cost. We apply principle of optimality for analysis. We first discuss the model with one failure mode, and then the model with several failure modes as the extension of the first one.

\section{EXTENDED BLOCK REPLACEMENT MODEL WITH ONE FAILURE MODE}

We discuss the block replacement model, in which after a preventive maintenance the system is not always as good as new. It is shown that there exists a finite and unique optimum policy under certain conditions, and also the relationship between the result of this section and the result obtained in the earlier contribution is discussed.

\section{Notation}

$\psi(\cdot \mid x), \quad$ any conditional function $\psi$ on the condition that a preventive maintenance is done by a unit aged $x$, where age $x$ is a random variable;

$r(t \mid x), \quad$ conditional failure rate for a unit when the unit has been operating during time interval $t$ after a preventive maintenance;

$G_{e}(x), \quad$ cumulative distribution function (cdf) of age $x$ of a unit for a preventive maintenance at acquisition time point;

$c_{a}(x), \quad$ cost suffered for each acquisition of a unit aged $x$;

$c_{m}$, cost suffered for each minimal repair;

R.A.I.R.O. Recherche opérationnelle/Operations Research 
$c_{s}$, cost suffered for the exchanged unit, which is still able to operate, at each preventive maintenance, i. e., suffered for cannibalization of a unit;

$k_{0}, \quad$ cost per unit time suffered for operating of a unit;

$\bar{\psi}, \quad 1-\psi ; \psi$ is any function;

$\alpha, \quad$ continuous (exponential) type discount rate; $\alpha>0$; a unit of cost is discounted $\exp (-\alpha t)$ after a time interval $t$

$o(h), \quad$ "function $f($.$) is said to be o(h)$ if $\lim _{h \rightarrow 0} f(h) / h=0$ " (see Ross [9], p. 117);

$T$, interval between preventive maintenances;

$T^{*}$, optimum interval between preventive maintenances minimizing the expected total discounted cost for an infinite time span;

$C_{u}(T ; t), \quad$ minimum expected total discounted cost for an infinite time span when a unit has been operating during time interval $t$ after a preventive maintenance and has not failed; $0 \leqq t \leqq T$;

$C_{d}(T ; t)$, minimum expected total discounted cost for an infinite time span when a minimal repair has started, of a unit that has been operating during time interval $t$ after a preventive maintenance and has failed; $0 \leqq t \leqq T$.

\section{Model and assumptions}

1. Consider a one-unit system, where the unit is maintained preventively and periodically at a prespecified time $k T(k=0,1,2, \ldots)$, and the unit is always made minimal repair when it fails.

2. The planning horizon is infinite.

3. System failure is revealed instanteneously.

4. The preventive maintenance and the minimal repair are made instantaneously.

5. The value of a unit is the highest at the acquisition time point, i. e., $c_{s}+c_{a}(x)>0$.

6. An interval between preventive maintenances is one cycle.

\section{Analysis and theorems}

Principle of optimality (see Bellman and Dreyfus [4], p. 15) is applied in the following discussion. 
Minimum expected total discounted $\operatorname{cost} C_{d}(T ; t)$ is obtained as follows:

$$
C_{d}(T ; t)=\int_{0}^{\infty}\left[c_{m}+C_{u}(T ; t \mid x)\right] d G_{e}(x)=c_{m}+C_{u}(T ; t) .
$$

Minimum expected total discounted cost $C_{u}(T ; t)$ is obtained as follows: We have the following functional equation for $0 \leqq t \leqq T$;

$$
C_{u}(T ; t)=\min \left\{\begin{array}{c}
L(t) \quad \text { for } 0 \leqq t \leqq T \\
A(t) \text { for } t=T
\end{array}\right.
$$

where :

$$
\begin{aligned}
& L(t)=\int_{0}^{\infty}\left[k_{0} \int_{0}^{d t} \exp (-\alpha \tau) d \tau+\right.\{1-r(t \mid x) d t+o(d t)\} \\
& \times C_{u}(T ; t+d t \mid x) \exp (-\alpha d t)+\{r(t \mid x) d t+o(d t)\} \\
&\left.\times C_{d}(T ; t+d t \mid x) \exp (-\alpha d t)\right] d G_{e}(x),
\end{aligned}
$$

and :

$$
A(t)=\int_{0}^{\infty}\left[c_{s}+c_{a}(x)+C_{u}(T ; 0 \mid x)\right] d G_{e}(x)
$$

Thus, for $0 \leqq t \leqq T$ we have that:

$$
\begin{aligned}
L(t)=C_{u}(T ; t)+\left[-\alpha C_{u}(T ; t)\right. & \left.+d C_{u}(T ; t) / d t\right] d t \\
& +\int_{0}^{\infty}\left[k_{0}+c_{m} r(t \mid x)+o(d t) / d t\right] d G_{e}(x) d t .
\end{aligned}
$$

Thus, from $C_{u}(T ; t)=L(t)$ :

$$
\begin{aligned}
C_{u}(T ; t)=\exp (\alpha t)\left[C_{u}(T ; 0)-\int_{0}^{\infty} \int_{0}^{t} \exp (-\alpha \tau)\left\{k_{0}\right.\right. & \\
& \left.\left.+c_{m} r(\tau \mid x)\right\} d \tau d G_{e}(x)\right],
\end{aligned}
$$

for $0 \leqq t \leqq T$. On the other hand, for $t=T$ :

$$
A(t)=C_{u}(T ; 0)+\int_{0}^{\infty}\left[c_{s}+c_{a}(x)\right] d G_{e}(x)
$$


Thus:

$$
C_{u}(T ; T)=C_{u}(T ; 0)+\int_{0}^{\infty}\left[c_{s}+c_{a}(x)\right] d G_{e}(x) .
$$

Thus, from $C_{u}(T ; T)$ evaluated at $t=T$ in the formula (6) and the formula (8), we obtain $C_{u}(T ; 0)$; that is:

$$
\begin{aligned}
C_{u}(T ; 0)=\int_{0}^{\infty}\left[\operatorname { e x p } ( - \alpha T ) \left\{c_{s}\right.\right. & \left.+c_{a}(x)\right\}+\int_{0}^{T} \exp (-\alpha t)\left\{k_{0}\right. \\
& \left.\left.+c_{m} r(t \mid x)\right\} d t\right] d G_{e}(x) /[1-\exp (-\alpha T)] .
\end{aligned}
$$

When $T \rightarrow \infty$, we have:

$$
C_{u}(\infty ; 0)=k_{0} / \alpha+c_{m} \int_{0}^{\infty} \int_{0}^{\infty} \exp (-\alpha t) r(t \mid x) d t d G_{e}(x)
$$

Differentiating the expected total discounted cost $C_{u}(T ; 0)$ with respect to $T$, setting it equal to zero and arranging it, we have $q_{u \alpha}(T)=0$, where:

$$
\begin{aligned}
q_{u \alpha}(T)=[1- & \exp (-\alpha T)] \int_{0}^{\infty}\left[-\alpha\left\{c_{s}+c_{a}(x)\right\}+k_{0}\right. \\
& \left.+c_{m} r(T \mid x)\right] d G_{e}(x)-\alpha \int_{0}^{\infty}\left[\exp (-\alpha T)\left\{c_{s}+c_{a}(x)\right\}\right. \\
& \left.+\int_{0}^{T} \exp (-\alpha t)\left\{k_{0}+c_{m} r(t \mid x)\right\} d t\right] d G_{e}(x),
\end{aligned}
$$

and:

$$
\begin{aligned}
q_{u \alpha}(\infty)=\int_{0}^{\infty}\left[-\alpha\left\{c_{s}+c_{a}(x)+c_{m} \int_{0}^{\infty} \exp (-\alpha t) r(t \mid x) d t\right\}\right. & \\
& \left.+c_{m} r(\infty \mid x)\right] d G_{e}(x)
\end{aligned}
$$

Here, we have the following theorem. The proof is given in Appendix 1.

THEOREM 1: If there exists the optimum interval $T^{*}$, which minimizes $C_{u}(T ; 0)$ in the formula (9), and at present a unit has been operating during time interval $t\left(0 \leqq t \leqq T^{*}\right)$ after a preventive maintenance, then the optimum policy is one that the unit is maintained preventively at $T^{*}$. 
Thus, we discuss the optimum interval $T^{*}$ which minimizes $C_{u}(T ; 0)$ in the formula (9). We have the following theorem on the sufficient condition for the existence of the optimum interval $T^{*}$. The proof is given in Appendix 2.

THEOREM 2: There exists at least an optimum interval $T^{*}\left(0<\mathrm{T}^{*} \leqq \infty\right)$ minimizing $C_{u}(T ; 0)$, and furthermore if $q_{u x}(\infty)>0$, then there exists at least a finite $T^{*}\left(0<T^{*}<\infty\right)$.

The above theorem shows that there is at least an optimum interval $T^{*}$, which is not necessarily unique, under a certain condition. Next, we have the following main theorem supposing the monotone property of failure rate, i. e., a strictly increasing or decreasing property. The proof is given in Appendix 3.

THEOREM 3:(1) If the failure rate $r(T \mid x)$ is strictly increasing with respect to $T$ and $q_{u x}(\infty)>0$, then there exists a finite and unique optimum interval $T^{*}\left(0<T^{*}<\infty\right)$ satisfying $q_{u \alpha}(T)=0$ and the corresponding expected total discounted cost is:

$$
C_{u}\left(T^{*} ; 0\right)=(1 / \alpha) \int_{0}^{\infty}\left[-\alpha\left\{c_{s}+c_{a}(x)\right\}+k_{0}+c_{m} r\left(T^{*} \mid x\right)\right] d G_{e}(x)
$$

(2) If the failure rate $r(T \mid x)$ is strictly increasing with respect to $T$ and $q_{u x}(\infty) \leqq 0$, or if the failure rate $r(T \mid x)$ is decreasing with respect to $T$, then the op timum interval is $T^{*} \rightarrow \infty$, i.e., a preventive maintenance is not made at all but a minimal repair is only made. The corresponding expected total discounted cost is given in the formula (10).

RemarKs: In this section, we have discussed the block replacement model in which after a preventive maintenance the system is not always as good as new with one failure mode, and have obtained three theorems on the optimum policy which minimizes the expected total discounted cost for an infinite time span. It has been shown that there exists a finite and unique optimum interval under certain conditions.

In particular, if $G_{e}(x) \equiv u(x-s)$, where $u(\cdot)$ is a unit function, $r(t \mid x) \equiv r(t+x), c_{s} \equiv 0$, and $k_{0} \equiv 0$, then we have:

$$
\lim _{\alpha \rightarrow 0} \alpha C_{u}(T ; 0)=\left[c_{a}(s)+c_{m} \int_{s}^{s+T} r(t) d t\right] / T,
$$

and this is equal to the expected cost per unit time in the steady-state for the block replacement model (periodic replacement model with minimal repair) discussed by Nakagawa [8]. 


\section{CASE WITH SEVERAL FAILURE MODES}

In this section, we extend the model in the preceding section to the model with several failure modes and use some non-linear costs.

\section{Notation}

Subscript $i$ index, which denotes failure mode $i(i=1,2, \ldots, n)$.

$r_{i}(t \mid x)$, conditional failure rate for failure mode $i$ when a unit has been operating during time interval $t$ after a preventive maintenance;

$a_{i}$, weight factor for failure mode $i ; a_{i} \geqq 0$;

$$
\sum_{i=1}^{n} a_{i}=1 \text {; }
$$

$v_{r}(t \mid x), \quad \sum_{i=1}^{n} a_{i} c_{m i} r_{i}(t \mid x)$

$c_{m i}, \quad$ cost suffered for each minimal repair for failure mode $i$;

$c_{s}(t \mid x), \quad$ conditional salvage cost when a unit has been operating during time interval $t$ after a preventive maintenance and has not failed;

$k_{0}(t \mid x)$, conditional operational cost when a unit has been operating during time interval $t$ after a preventive maintenance and has not failed;

$C_{F u}(T ; t)$, minimum expected total discounted cost for an infinite time span when a unit has been operating during time interval $t$ after a preventive maintenance and has not failed; $0 \leqq t \leqq T$.

For the others, see Notation in Section 2.

\section{Assumptions}

1. The lifetime for each unit obeys an arbitrary mixture distribution with $n$ failure modes.

2. $c_{s}(t \mid x)+c_{a}(x)>0$, i. e., the value of a unit is the highest at the acquisition time point.

3. When $d v_{r}(t \mid x) / d t>0$, then $d c_{s}(t \mid x) / d t \leqq 0$ :

$$
d^{2} c_{s}(t \mid x) / d t^{2} \geqq 0 \quad \text { and } \quad d k_{0}(t \mid x) / d t \geqq 0 .
$$

When:

$$
\begin{array}{rlrl}
d v_{r}(t \mid x) / d t \leqq 0 & \text { then } \quad d c_{s}(t \mid x) / d t \geqq 0, \\
d^{2} c_{s}(t \mid x) / d t^{2} & \leqq 0 & \text { and } \quad & d k_{0}(t \mid x) / d t \leqq 0 .
\end{array}
$$

For the others, See Model and assumptions in Section 2. 


\section{Analysis and theorem}

We obtain the following quantities in a similar fashion of the analysis in Section 2:

For $0 \leqq t \leqq T$ :

$$
\begin{array}{r}
C_{F u}(T ; t)=\exp (\alpha t)\left[C_{F u}(T ; 0)-\int_{0}^{\infty} \int_{0}^{t} \exp (-\alpha \tau)\left\{k_{0}(\tau \mid x)\right.\right. \\
\left.\left.+v_{r}(\tau \mid x)\right\}\right] d \tau d G_{e}(x),
\end{array}
$$

where:

$$
\begin{aligned}
C_{F u}(T ; 0)=\int_{0}^{\infty} \mid & \exp (-\alpha T)\left\{c_{s}(T \mid x)+c_{a}(x)\right\} \\
& \left.+\int_{0}^{T} \exp (-\alpha t)\left\{k_{0}(t \mid x)+v_{r}(t \mid x)\right\} d t\right] d G_{e}(x)
\end{aligned}
$$

and:

$$
C_{F u}(\infty ; 0)=\int_{0}^{\infty} \int_{0}^{\infty} \exp (-\alpha t)\left[k_{0}(t \mid x)+v_{r}(t \mid x)\right] d t d G_{e}(x)
$$

Furthermore:

$$
\begin{aligned}
q_{\mathrm{Fux}}(T)=[1-\exp (-\alpha T)] & \int_{0}^{\infty}\left[-\alpha\left\{c_{s}(T \mid x)+c_{a}(x)\right\}\right. \\
+ & \left.d c_{s}(T \mid x) / d T+k_{0}(T \mid x)+v_{r}(T \mid x)\right] d G_{e}(x) \\
-\alpha & \int_{0}^{\infty}\left[\exp (-\alpha \mathrm{T})\left\{c_{s}(T \mid x)+c_{a}(x)\right\}\right. \\
& \left.+\int_{0}^{T} \exp (-\alpha t)\left\{k_{0}(t \mid x)+v_{r}(t \mid x)\right\} d t\right] d G_{e}(x),
\end{aligned}
$$

and:

$$
\begin{aligned}
& q_{F u x}(\infty)=\int_{0}^{\infty}[-\alpha\left\{c_{s}(\infty \mid x)+c_{a}(x)+\int_{0}^{\infty} \exp (-\alpha t)\left\{k_{0}(t \mid x)\right.\right. \\
&\left.\left.+v_{r}(t \mid x)\right\} d t\right\}+\lim _{T \rightarrow \infty} d c_{s}(T \mid x) / d T \\
&+k_{0}(\infty \mid x) \\
&\left.+v_{r}(\infty \mid x)\right] d G_{e}(x) .
\end{aligned}
$$


Thus, theorems corresponding to Theorems 1-3 are obtained in similar fashions of their proofs. For exemple, the corresponding theorem to Theorem 3 is presented as follows:

THEOREM 4: (1) If $v_{r}(T \mid x)$ is strictly increasing with respect to $T$ and $q_{F u u}(\infty)>0$, then there exists a finite and unique optimum interval $T^{*}\left(0<T^{*}<\infty\right)$ satisfying $q_{F u \alpha}(T)=0$ and the corresponding expected total discounted cost is:

$$
\begin{aligned}
C_{F u}\left(T^{*} ; 0\right)=(1 / \alpha) \int_{0}^{\infty}\left[-\alpha\left\{c_{s}\left(T^{*} \mid x\right)+c_{a}(x)\right\}\right. \\
\left.+d c_{s}(T \mid x) /\left.d T\right|_{T=t^{*}}+k_{0}\left(T^{*} \mid x\right)+v_{r}\left(T^{*} \mid x\right)\right] d G_{e}(x),
\end{aligned}
$$

where $d c_{s}(T \mid x) /\left.d T\right|_{T=t^{*}}$ implies $d c_{s}(T \mid x) / d T$ evaluated at $T=T^{*}$.

(2) If $v_{r}(T \mid x)$ is strictly increasing with respect to $T$ and $q_{F u x}(\infty) \leqq 0$, or if $v_{r}(T \mid x)$ is decreasing with respect to $T$, then the optimum interval is $T^{*} \rightarrow \infty$, i. e., a preventive maintenance is not made at all but a minimal repair is only made. The corresponding expected total discounted cost is given in the formula (17).

REMARKS: We have discussed the block replacement model with several failure modes, a non-linear salvage cost, and a non-linear operational cost. This model is an extension of the model in Section 2.

\section{CONCLUDING REMARKS}

In this paper, we have treated the block replacement model with minimal repair. In Section 2, we have discussed the block replacement model with one failure mode, in which after a preventive maintenance the system is not always as good as new. In Section 3, we have extended the model in Section 2 to the model with several failure modes, a non-linear salvage cost, and a non-linear operational cost.

As a criterion, we have applied the expected total discounted cost for an infinite time span, especially with a continuous type discount rate, and obtained the optimum interval between preventive maintenances minimizing that expected cost. It has been shown that there exists a finite and unique optimum interval under certain conditions. We have applied principle of optimality to analyze these models.

\section{APPENDIX}

\section{THE PROOF OF THEOREM 1}

If there exists the optimum interval $T^{*}$ minimizing $\mathrm{C}_{u}(T ; 0)$ and at present the unit has been operating during time interval $t\left(0 \leqq t \leqq T^{*}\right)$ after a preventive vol. $18, \mathrm{n}^{\circ} 1$, février 1984 
maintenance, then $T^{*}$ minimizes $C_{u}(T ; t)$ in the formula $(6)$ for any $t\left(0 \leqq t \leqq T^{*}\right)$ from substituting $C_{u}(T ; 0)$ into $C_{u}(T ; t)$.

Q.E.D.

\section{THE PROOF OF THEOREM 2}

Differentiating $\log C_{u}(T ; 0)$ with respect to $T$, we have:

$\left[d \log C_{u}(T ; 0)\right] /[d t]$

$$
\begin{gathered}
=\left[\exp (-\alpha T) /\left[\int _ { 0 } ^ { \infty } \left\{\exp (-\alpha T)\left(c_{s}+c_{a}(x)\right)+\int_{0}^{T} \exp (-\alpha t)\left(k_{0}\right.\right.\right.\right. \\
\left.\left.\left.\left.+c_{m} r(t \mid x)\right) d t\right\} d G_{e}(x)\{1-\exp (-\alpha T)\}\right]\right][[1-\exp (-\alpha T)] \\
\quad \times \int_{0}^{\infty}\left[-\alpha\left\{c_{s}+c_{a}(x)\right\}+k_{0}+c_{m} r(T \mid x)\right] d G_{e}(x) \\
-\alpha \int_{0}^{\infty}\left[\exp (-\alpha T)\left\{c_{s}+c_{a}(x)\right\}+\int_{0}^{T} \exp (-\alpha t)\left\{k_{0}\right.\right. \\
\left.\left.\left.+c_{m} r(t \mid x)\right\} d t\right] d G_{e}(x)\right] .
\end{gathered}
$$

For small $T$ :

$\left[d \log C_{u}(T ; 0)\right] /[d t]$

$$
\begin{array}{r}
\simeq\left[\exp (-\alpha T) /\left[\int _ { 0 } ^ { \infty } \left\{\exp (-\alpha T)\left(c_{s}+c_{a}(x)\right)+\int_{0}^{T} \exp (-\alpha t)\left(k_{0}\right.\right.\right.\right. \\
\left.\left.\left.\left.+c_{m} r(t \mid x)\right) d t\right\} d G_{e}(x)\{1-\exp (-\alpha T)\}\right]\right]\left[-\alpha \int_{0}^{\infty}\left\{c_{s}\right.\right. \\
\left.\left.+c_{a}(x)\right\} d G_{e}(x)\right]<0,
\end{array}
$$

which implies that there exists at least an optimum interval $T^{*}\left(0<T^{*} \leqq \infty\right)$ minimizing $C_{u}(T ; 0)$. Furthermore, for large $T$ :

$\left[d \log C_{u}(T ; 0)\right] /[d T]$

$$
\simeq\left[\exp (-\alpha T) /\left[\int _ { 0 } ^ { \infty } \left\{\exp (-\alpha T)\left(c_{s}+c_{a}(x)\right)+\int_{0}^{T} \exp (-\alpha T)\left(k_{0}\right.\right.\right.\right.
$$




$$
\begin{aligned}
& \left.\left.\left.\left.+c_{m} r(t \mid x)\right) d t\right\} d G_{e}(x)\{1-\exp (-\alpha T)\}\right]\right]\left[\int _ { 0 } ^ { \infty } \left[-\alpha\left\{c_{s}\right.\right.\right. \\
& \left.\left.\left.+c_{a}(x)+c_{m} \int_{0}^{\infty} \exp (-\alpha t) r(t \mid x) d t\right\}+c_{m} r(\infty \mid x)\right] d G_{e}(x)\right]
\end{aligned}
$$

which implies that if $q_{u \alpha}(\infty)>0$, then there exists at least a finite $T^{*}\left(0<T^{*}<\infty\right)$.

Q.E.D.

\section{THE PROOF OF THEOREM 3}

We have:

$$
q_{u \alpha}^{\prime}(T)=[1-\exp (-\alpha T)] c_{m} \int_{0}^{\infty}(d r(T \mid x) / d t) d G_{e}(x)
$$

First, we assume the case that the failure rate is strictly increasing. Thus, we have that $q_{u \alpha}^{\prime}(T)>0$, i. e., $q_{u \alpha}(T)$ is strictly increasing.

If $q_{u \alpha}(\infty)>0$, then there exists a finite and unique $T^{*}\left(0<T^{*}<\infty\right)$ which minimizes $C_{u}(T ; 0)$ as a finite and unique solution to $q_{u \alpha}(T)=0$, since $q_{u \alpha}(0)<0, q_{u \alpha}(T)$ is strictly increasing and continuous. Substituting the relation of $q_{u \alpha}\left(T^{*}\right)=0$ into $C_{u}\left(T^{*} ; 0\right)$ in the formula (9) yields the formula (13).

If $q_{u \alpha}(\infty) \leqq 0$, then for any non-negative $T, C_{u}^{\prime}(T ; 0) \leqq 0$ and thus $C_{u}(T ; 0)$ is a strictly decreasing function. Thus, $T^{*} \rightarrow \infty$.

Secondly, we assume the case that the failure rate is decreasing. Thus, we have that $q_{u \alpha}^{\prime}(T) \leqq 0$, i. e., $q_{u \alpha}(T)$ is decreasing. Since $q_{u \alpha}(0)<0$, for any non-negative $T, C_{u}^{\prime}(T ; 0)<0$ and thus $C_{u}(T ; 0)$ is a strictly decreasing function. Thus, $T^{*} \rightarrow \infty$.

Q.E.D.

\section{REFERENCES}

1. R. E. Barlow and L. C. Hunter, Optimum Preventive Maintenance Policies, Operations Research, Vol. 8, 1960, pp. 90-100.

2. R. E. Barlow and F. Proschan, Mathematical Theory of Reliability, Wiley, New York, 1965.

3. R. E. Barlow and F. Proschan, Statistical Theory of Reliability and Life Testing: Probability Models, Holt, Rinehart and Winston, New York, 1975.

4. R. E. Bellman and S. E. Dreyfus, Applied Dynamic Programming, Princeton University Press, Princeton, New Jersey, 1962.

5. M. Berg and B. Epstein, A Modified Block Replacement Policy, Naval Research Logistics Quaterly, Vol. 23, 1976, pp. 15-24.

vol. $18, \mathrm{n}^{\circ} 1$, février 1984 
6. M. BERG and B. EPSTEIN, A Note on a Modified Block Replacement Policy for Units with Increasing Marginal Running Costs, Naval Research Logistics Quarterly, Vol. 26, 1979, pp. 157-160.

7. N. KaIo and S. Osaki, Optimum Age Replacement Policy with Two Failures Modes, R.A.I.R.O., Vol. 13, 1979, pp. 205-209.

8. T. Nakagawa, Optimum Replacement Policies for a Used Unit, J. Operations Res. Soc. of Japan, Vol. 22, 1979, pp. 338-346.

9. S. M. Ross, Introduction to Probability Models, Academic Press, New York, 1972. 